\title{
Ultrasonic Methods of the Cells Separation in Human Blood
}

\author{
A. WŁOCH*, H. CzYŻ And T. JAsińsKi \\ Faculty of Mathematics and Applied Physics, Rzeszów University of Technology, Rzeszów, Poland \\ This work deals with the problem of ultrasound in the context of biomedical and clinical applications. Ultra- \\ sonic waves can be used for the separation of cells in human blood. Under the influence of ultrasonic waves cells \\ included in the liquid experience a certain characteristic displacement was referred to as drift. Presented work \\ suggests that only radiation pressure should be taken into account as a mechanism of drifting in this situation. It \\ has been demonstrated that the growth of cells concentration around the points of stable equilibrium is exponential. \\ The time constant of this growth was estimated and the formulae which determine the time needed to obtain the \\ assumed concentration increase have been derived. The theoretical studies of acoustic agglomeration of particles \\ in the liquid have become a new focus in the ultrasonic research.
}

DOI: $10.12693 /$ APhysPolA.128.234

PACS: $43.35 .+\mathrm{d}, 47.35 . \mathrm{Rs}, 62.60 .+\mathrm{v}, 83.80 . \mathrm{Hj}, 87.50 . \mathrm{Y}_{-}$

\section{Introduction}

The influence of ultrasonic waves on the diffracting phase is interesting by itself as one of the fundamental physical effects of ultrasound. The physical properties of ultrasound and their complex interactions with human tissues led to the fact that they are an important tool in medicine [1]. In this work we explore physical and engineering principles of acoustics and using ultrasound for medical applications [2-4]. The interaction of ultrasonic waves with dispersions can be used for separating the cells from the liquid. These mechanisms are very complex processes and their theoretical descriptions are not completely established. Many mechanisms have been described to explain a motion (drift) of particles in an ultrasonic field. This motion of particles (cells) in an ultrasonic field consists of monotonically approaching to the stable equilibrium point or quasi periodical vibration with amplitude damping. The ultrasonic wave accelerates the transport of cells to the minimal zone of potential drift force. This kinetics process depends on the ultrasonic field, the liquid and cells parameters. We consider the question of time variation of particle concentration under the influence of drift force. We show that the concentration at the points of stable equilibrium increases exponentially.

\section{The theory outline}

Under the influence of the standing wave ultrasonic field the cells are directed to the flat of minimum potential of drift forces. A few mechanisms responsible for cells motion in the standing wave field are proposed in the literature [5]. The drift forces determine the result of interaction between a dispersed phase and a medium vibration. The review of the drift forces should begin

\footnotetext{
*corresponding author; e-mail: awloch@prz.edu.pl
}

with the so-called radiation drift related to the radiation pressure. Calculating the difference between the momentum of the incident wave and that of a wave scattered on a particle gave an expression of the radiation drift force $F_{D R}$, the $R$-type drift in short. The asymmetry drift results only from the fact that the motion of the medium in the standing wave is asymmetric - the asymmetry of medium oscillations resulting from the standing wave amplitude dependence on position. That is the reason why we use the index $\mathrm{A}$ for this force $F_{\mathrm{DA}}$, the A-type drift in short. Another type of a drift, the L-type drift in short, is connected with periodic changes in the viscosity of the medium, which in turn result from periodic changes in temperature in a standing wave field.

The drift forces show the same dependence on the position. It appears that, irrespective of the mechanism of the occurrence of such forces, they can be described by formula $F_{D}(x)=F_{0} \sin (2 k x)$. The potential related to their action has the form $U_{D}(x)=F_{0}(2 k)^{-1} \cos (2 k x)$. The position of the potential minima depends on the sign of the constant $F_{0}$, describing the maximum value of the drift force. For $F_{0}>0$ the minima of potential coincide with the value $2 k x=(2 n+1) \pi$, giving the equation of the planes of antinodes. In turn, for $F_{0}<0$ the drift forces gather cells at the nodes of the standing waves. It is clear that the sign of the constant $F_{0}$ has no effect on the kinetics of the process of cell transport. Although there exists an extensive literature on the interaction of ultrasonic waves with particles, all these works deal with the one dimensional case.

\section{Results and discussion}

For all types of drift, irrespectively of the considered mechanism of the phenomenon, the equation of motion of particle is of the form

$$
m_{p} \ddot{x}+6 \pi \eta r_{p} \dot{x}=F_{D} \sin (2 k x),
$$

where $m_{p}$ - mass of a particle, $r_{p}$ - particle radius, $x-$ position measured along wave propagation direction, $\eta-$ kinematic viscosity of the liquid, $k$ - wave number, $F_{D}$ 
denotes the amplitude of the drift force. Dividing Eq. (1) by $m_{p}$, we obtain $A_{D}$, which denotes the maximum value of the drift force acceleration

$$
\ddot{x}+\tau^{-1} \dot{x}=A_{D} \sin (2 k x),
$$

where the relaxation time

$$
\tau=\frac{2 \rho_{p} r_{p}^{2}}{9 \eta}
$$

the value of which depends on the parameters of cells and the coefficient of the Stokes resistance connected to the viscosity of the medium, characterizes the effect of the resistance on the motion.

For all types of the drift the formulae of the drift force acceleration are following: for A-type drift:

$$
A_{\mathrm{DA}}=-\frac{1}{2} \rho_{g}^{-1} k \mu_{p}^{2} \bar{E}
$$

for $R$-type drift:

$$
A_{D R}=2 k \rho_{p}^{-1} \mu_{g}^{2} \bar{E}
$$

for L-type drift:

$$
A_{\mathrm{DL}}=\frac{9}{4}(\kappa-3) \eta r_{p}^{-2}\left(\rho_{p} \rho_{g} c\right)^{-1} \mu_{g}^{2} \bar{E}
$$

where the entertainment coefficient

$$
\mu_{p}=\frac{1}{\sqrt{1+\omega^{2} \tau^{2}}}
$$

is the flow-around coefficient

$$
\mu_{g}=\frac{\omega \tau}{\sqrt{1+\omega^{2} \tau^{2}}} \text {. }
$$

The entertainment coefficient (7) is the coefficient of proportionality between the vibration amplitudes of the medium and the cell, along with the flow coefficient (8), it satisfies the relation $\mu_{p}^{2}+\mu_{g}^{2}=1$.

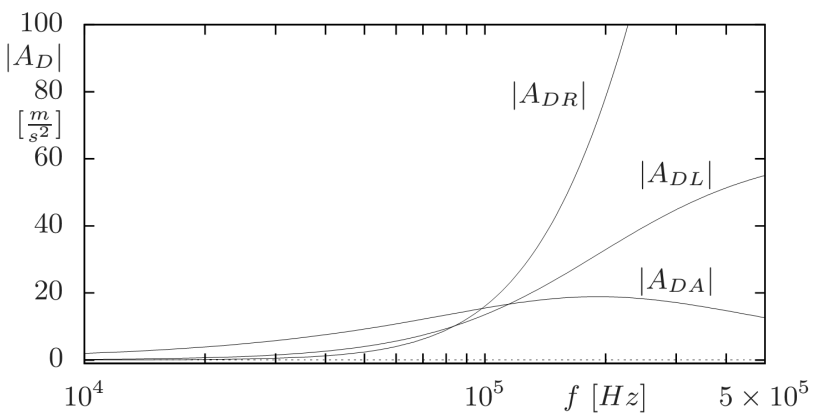

Fig. 1. Absolute values of the drifts force acceleration as a function of frequency.

Figure 1 presents plots of $A_{D}$ versus wave frequency for all types of drift. In order to calculate values for the graph we have adopted the following values for the parameters describing the cells, the fluid (plasma) and the acoustic field: density of red blood cells $\rho_{p}=$ $1080 \mathrm{~kg} \mathrm{~m}^{-3}, r_{p}=4 \times 10^{-6} \mathrm{~m}$, density of plasma $\rho_{g}=1070 \mathrm{~kg} \mathrm{~m}^{-3}, c=1550 \mathrm{~m} \mathrm{~s}^{-1}, \kappa=1.33, \eta=4.62 \times$ $10^{-3} \mathrm{~N} \mathrm{~s} \mathrm{~m}^{-2}$, average energy density $\bar{E}=100 \mathrm{~J} \mathrm{~m}^{-3}$, frequencies: $10^{5}$ to $10^{7} \mathrm{~Hz}$. It can be observed that the effect of the drift force is much greater than that of gravity $\left(g \approx 10 \mathrm{~m} \mathrm{~s}^{-2}\right)$. The analysis of the plots indicates that the $R$-type drift is the strongest, it dominates. The $R$-type drift should be taken into account as a mechanism of separating the cells from the plasma.

\section{The density distribution}

Denoting by $N=N(x, t)$ the number of particles per unit volume in a plane described by coordinate $x$ at instant $t$ the equation of conservation of the number of particles (the continuity equation) is then

$$
\frac{\partial}{\partial x}\left(N u_{p}\right)+\frac{\partial}{\partial t} N=0
$$

where $\dot{x} \equiv u_{p}=V_{D R} \sin (2 k x)$ and $V_{D R}=\tau A_{D R}$ is the drift velocity amplitude. The values of $V_{D R}$ depended strongly on the particle radius and frequency via the coefficients $\mu_{p}, \mu_{g}$ which define $F_{D R}$. After some algebra we obtain for the radiation drift

$$
V_{D R}=2 \frac{\rho_{g}}{\rho_{p}}(\omega \tau)^{3}\left[1+(\omega \tau)^{2}\right]^{-1} \frac{\bar{E}}{R}
$$

where $R$ - acoustic impedance, in acoustics is the resistance of the medium, expanding wave ultrasound.

We assume that at the instant $t=0$, the distribution function was constant and equal to $N_{0}$. Thus we have, after some manipulations

$$
N(x, t)=\frac{N_{0}}{\sin ^{2}(k x) \exp (-\delta t)+\cos ^{2}(k x) \exp (\delta t)},
$$

where $\delta=2 k V_{D R}$.

The quantity $\delta^{-1}$ is thus the time constant of the exponential growth of the concentration in the regions of stable equilibrium.

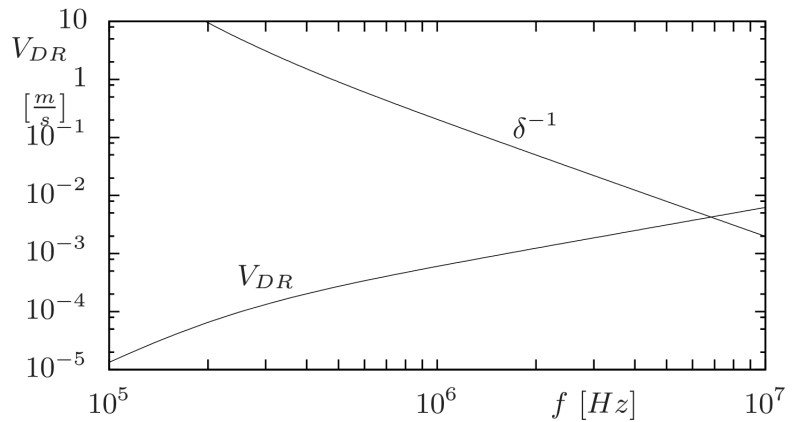

Fig. 2. The $R$-type drift velocity amplitude as a function of frequency.

Figure 2 presents the plot of $V_{D R}$ and $\delta^{-1}$ versus frequency. The changes of the concentration of the cells as a function of position and time on the basis of formula (11) is shown in Fig. 3. The white color indicates the maximal concentration, the black color - the minimal concentration. The analysis of these graphs shows that multiple increase of the concentration takes place within a few seconds. As you can see at the node of the standing wave the concentration decreases as $N_{0} \mathrm{e}^{-\delta t}$, while it increases at antinodes as $N_{0} \mathrm{e}^{\delta t}$. 

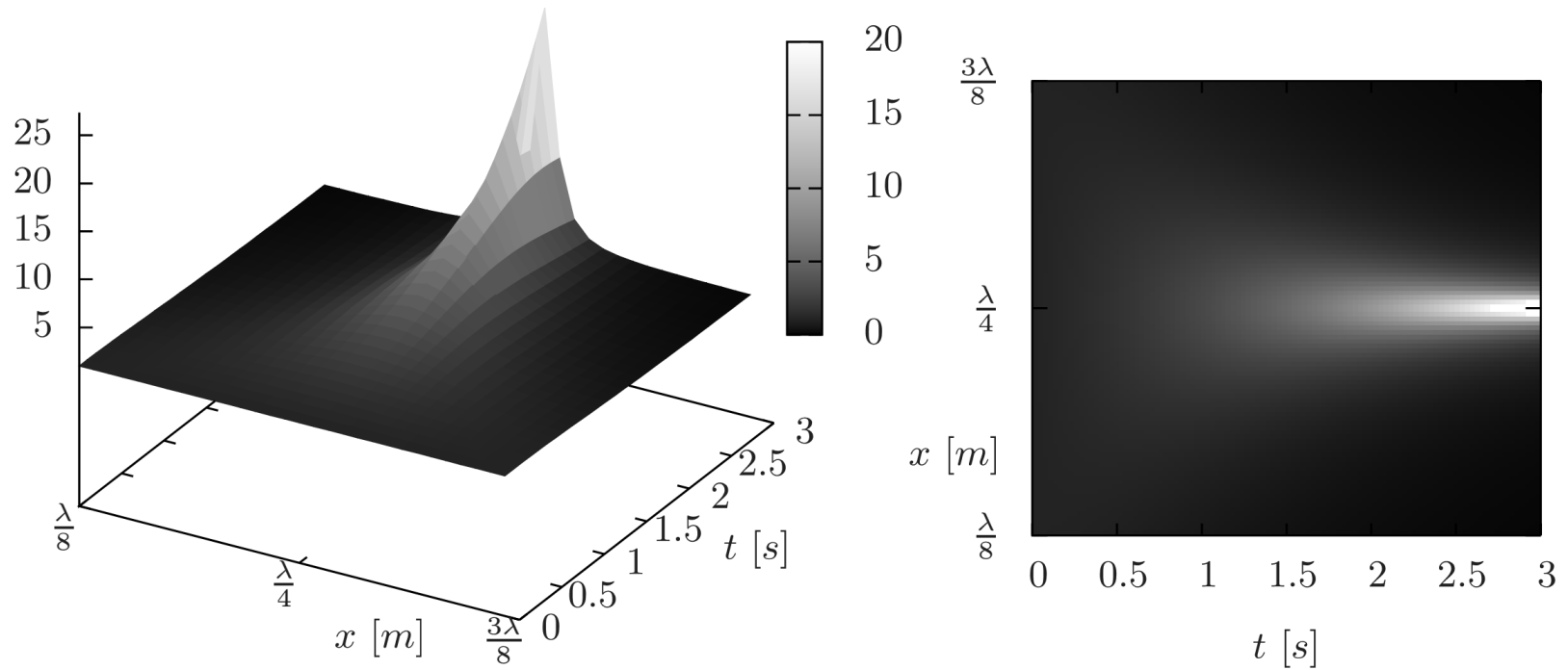

Fig. 3. The concentration of the cells as a function of position and time for $f=5 \times 10^{5}[\mathrm{~Hz}]$.

\section{Conclusions}

In conclusion, it can be stated that the $R$-type drift force directs cells to the antinodes of the standing wave and acts in the most efficient way on cells with radii of the order of $10^{-6} \mathrm{~m}[6]$. For the above listed values of acoustic wave parameters, the $R$-type drift effectively gathers the cells around the equilibrium positions in the time shorter than $3 \mathrm{~s}$.

\section{References}

[1] E. Benes, M. Gröschl, S. Radel, C. Hauser, H. Böhm, H. Nowotny, in: Proc. 2nd Congress of Alps-Adria Acoustics Association and 1st Congress of Acoustical Society of Croatia, 2005, p. 23.

[2] F. Trampler, D. Schwartz, W. Mayr, E. Benes, in: Proc. WCU 97 - World Congress on Ultrasonics, Yokohama 1997, p. 320.
[3] P. Sarvazyan, O.V. Rudenko, W.L. Nyborg, Ultrasound Med. Biol. 36, 1379 (2010).

[4] A. Haake, A. Neild, D.H. Kim, J.E. Ihm, Y. Sun, J. Dual, B.K. Ju, Ultrasound Med. Biol. 31, 857 (2005).

[5] H. Czyż, in: Proc. 4th Europ. Conf. on Acoustics, vol. I, Eds.: A. Alippi, G.B. Cannelli, Italian Research Council, Roma 1998, p. 125.

[6] H. Mitome, T. Kozuka, T. Tuziuti, M. Gröschl, B. Handl, E. Benes, in: Proc. WCU 97 - World Congress on Ultrasonics, Yokohama 1997, p. 200. 